\title{
Functional Implications for Modulating Neuropeptide Y Gene Expression in the Dorsomedial Hypothalamus
}

\author{
Nicole R. Kinzeler and Kristin S. Edwards \\ Department of Psychology, Behavioral Neuroscience Graduate Program, Ohio State University, Columbus, Ohio 43210 \\ Review of Yang et al. (http://www.jneurosci.org/cgi/content/full/29/1/179)
}

Neuropeptide $\mathrm{Y}$ is a potent orexigenic hypothalamic peptide. Extensive research on the role of neuropeptide $\mathrm{Y}$ in feeding and the regulation of energy balance has provided a better understanding of the mechanisms and circuitry driving food intake, and has recently lead to the development of potential treatments for obesity and diabetes. Previous studies have demonstrated that acute systemic and central infusion of neuropeptide $\mathrm{Y}$ induces hyperphagia, and chronic administration leads to obesity (Stanley et al., 1986). Antagonizing neuropeptide Y receptors decreases food intake (Gehlert, 1999). Currently, clinical trials are underway in obese patients to examine the therapeutic efficacy of systemically blocking neuropeptide $\mathrm{Y}$ on food intake and body weight (Erondu et al., 2006; MacNeil, 2007).

Neurons containing neuropeptide Y are primarily located in areas involved in homeostatic functions such as the arcuate nucleus and dorsomedial hypothalamus. Whereas the role of neuropeptide $\mathrm{Y}$ in integrating neural and hormonal signals in the arcuate nucleus has been relatively well characterized, its role in the dorso-

\footnotetext{
Received May 8, 2009; accepted May 8, 2009.

We thank Dr. Courtney DeVries and Dr. Susan Travers for helpful comments regarding this manuscript.

Correspondence should be addressed to either of the following: Nicole R. Kinzeler, 305 W. 12th Avenue, Postle Hall, Room 4169, Columbus, $\mathrm{OH}$ 43210, E-mail: kinzeler.2@osu.edu; or Kristin S. Edwards, 1835 Neil Avenue, Psychology Building, Room 125, Columbus, $\mathrm{OH}$ 43210, E-mail: edwards.719@osu.edu.

DOI:10.1523/JNEUROSCI.1647-09.2009

Copyright $\odot 2009$ Society for Neuroscience $\quad 0270-6474 / 09 / 297389-03 \$ 15.00 / 0$
}

medial hypothalamus has not been as extensively studied. It is known that dorsomedial hypothalamic neuropeptide $\mathrm{Y}$ is enhanced in rodent models of obesity and with chronic food restriction, suggesting an important role for neuropeptide $\mathrm{Y}$ in the long-term control of food intake (Bi et al., 2003). Therefore, Yang et al. (2009) further characterized neuropeptide $\mathrm{Y}$ in the dorsomedial hypothalamus using adeno-associated virus to alter expression of neuropeptide $\mathrm{Y}$, and examined the long-term consequences of doing so with molecular, histological, and behavioral techniques.

Adeno-associated virus is a nonpathogenic, noncytotoxic retrovirus that delivers genes of interest to alter expression of proteins in the targeted area. This technique is particularly advantageous for these experiments because of its longlasting effects. Previously, the modulatory role of neuropeptides on feeding was studied using repeated systemic or central infusions that had effects for only a few hours. Although such studies have greatly contributed to our understanding of the mechanisms underlying energy homeostasis, long intervals of time are ideal to evaluate weight gain/loss over a more externally valid time course. In the present study, effects of altering neuropeptide $\mathrm{Y}$ expression on food intake and body weight lasted at least 6 months (Yang et al., 2009), providing an excellent animal model to study obesity.

First, Yang et al. (2009) generated an adeno-associated virus-mediated expres- sion vector to overexpress neuropeptide $\mathrm{Y}$ mRNA in the dorsomedial hypothalamus of lean Sprague Dawley rats [Yang et al. (2009), their Fig. $1 A$ ]. The vector was also tagged with green fluorescent protein to allow clear visualization of neurons overexpressing neuropeptide Y. A separate group of animals was injected with a control vector containing only green fluorescent protein. Western blot and in situ hybridization confirmed that the experimental procedure increased neuropeptide Y gene expression only in the targeted dorsomedial hypothalamus [Yang et al. (2009), their Fig. 1C,D]. Animals that overexpressed neuropeptide $\mathrm{Y}$ gained significantly more weight than controls [Yang et al. (2009), their Fig. 1 F]. The increase in body weight could be at least partly attributable to higher energy intake of both standard (weeks 1-4) and palatable, high-fat diet (weeks 5-12) [Yang et al. (2009), their Fig. 1G].

Yang et al. (2009) next examined the functional consequences of knocking down neuropeptide $\mathrm{Y}$ expression in the dorsomedial hypothalamus in a rodent model of obesity, by using the adenoassociated virus to express a short hairpin RNA (shRNA) targeting neuropeptide Y in Otsuka Long-Evans Tokushima Fatty (OLETF) rats. OLETF rats are particularly advantageous for the current study because they show blunted cholecystokinin satiety signaling and elevated neuropeptide Y mRNA expression during development, leading to hyperphagia and eventual obesity and diabetes. The authors 
compared OLETF rats receiving neuropeptide Y knockdown to two different control groups that received only the green florescent protein controlled vector: obese OLETF controls and lean LongEvans Tokushima Otsuka (LETO) rats. Only the neuropeptide Y knockdown animals showed a significant reduction of neuropeptide $\mathrm{Y}$ mRNA and protein levels in the dorsomedial hypothalamus [Yang et al. (2009), their Fig. 4]. As expected, the lean controls showed the lowest weight gain and energy intake, whereas the obese controls had the largest weight gain and intake. Knocking down neuropeptide $\mathrm{Y}$ in OLETF rats resulted in a significant reduction of body weight and food intake compared with obese controls [Yang et al. (2009), their Fig. 5A,B]. The normalization of feeding in the treated obese rats was associated with a reduction in diabetes risk factors, including blood glucose levels, plasma insulin, and body fat content [Yang et al. (2009), their Fig. 6].

Examination of the pattern of $24 \mathrm{~h}$ food intake after neuropeptide Y knockdown revealed that the reduction in feeding can be attributed to decreased meal size during the dark cycle [Yang et al. (2009), their Fig. 5C], suggesting modulation of within-meal satiety signaling. The dorsal vagal complex in the brainstem receives gastrointestinal satiety signals from peptide hormones, such as cholecystokinin, that reduce meal size. Yang et al. (2009) wanted to establish whether dorsomedial hypothalamic neuropeptide Y modulates cholecystokinin signaling in the brainstem. First, they found that knocking down neuropeptide $\mathrm{Y}$ in the dorsomedial hypothalamus decreased ipsilateral neuropeptide $\mathrm{Y}$ fiber staining in the dorsal vagal complex, suggesting that neuropeptide Y projections from the dorsomedial hypothalamus to the brainstem were disrupted [Yang et al. (2009), their Fig. 7]. Consequently, neuropeptide Y transmission was decreased, therefore enhancing the feeding inhibitory response to peripheral administration of cholecystokinin [Yang et al. (2009), their Fig. 8]. These results reveal potential mechanisms by which knocking down neuropeptide $\mathrm{Y}$ in the dorsomedial hypothalamus could attenuate hyperphagia and weight gain by enhancing satiety signals in the brainstem.

The paper by Yang et al. (2009) provides a greater understanding of the functional role of dorsomedial hypothalamic neuropeptide $\mathrm{Y}$ by using the adenoassociated virus to examine the long-term effects of altering neuropeptide $\mathrm{Y}$ expression on food intake and energy balance.
Their experimental approach is valuable because it allows chronic modification of neuropeptide Y gene expression, without the potential confounding issue of neuropeptide $\mathrm{Y}$ modulation during early development. The study is among the first to delineate a role for neuropeptide $\mathrm{Y}$ in the dorsomedial hypothalamus in feeding behavior. However, although these experiments provide interesting and significant contributions, there are some points of clarification, as well as future directions to consider for subsequent studies.

The first issue needing clarification regards the type of chow used in their behavioral measures. In the experiment examining the consequences of neuropeptide $\mathrm{Y}$ overexpression, both a standard rodent chow and a palatable high fat diet were used. However, when evaluating the effects of knocking down neuropeptide $\mathrm{Y}$ on feeding, Yang et al. (2009) only used standard chow. The authors did not provide a reason for this inconsistency. Looking at both standard and high-fat intake is important in determining whether neuropeptide $\mathrm{Y}$ increases intake by altering energy or reward signaling. Previous studies have revealed a preferential role for neuropeptide $\mathrm{Y}$ in the maintenance of energy homeostasis. Rats receiving neuropeptide $\mathrm{Y}$ infusion into the paraventricular nucleus consumed a higher proportion of calories from standard chow than from a sucrose solution, compared with those infused with saline (Giraudo et al., 1999). However, the present study demonstrated that overexpressing neuropeptide $\mathrm{Y}$ in the dorsomedial hypothalamus significantly increased intake of both diets, signifying that neuropeptide $\mathrm{Y}$ stimulates feeding based on the caloric value and the rewarding properties of the food. However, it is likely that knocking down neuropeptide $\mathrm{Y}$ would differentially alter intake of the two diets. OLETF rats have altered central gustatory processing of sugars and heightened preference for palatable foods (Kovacs and Hajnal, 2008); therefore, decreased hunger from enhanced satiety signaling after neuropeptide Y knockdown may be sufficient to reduce intake of a standard, but not a palatable diet. Because of this possibility, subsequent experiments should clarify the effects of both diet types.

Yang et al. (2009) attribute the increase in weight gain after neuropeptide Y overexpression to higher food intake. However, it will be important for future research to empirically examine other potential explanations for changes in body mass, such as changes in energy ex- penditure, thermoregulation, metabolic efficiency, and general activity levels. Neuropeptide Y plays a role in sugarinduced changes in energy expenditure (Levine et al., 2003). Additional exploration into other explanations for weight gain/loss would enhance our understanding of the role of dorsomedial hypothalamic neuropeptide $\mathrm{Y}$ in feeding behaviors.

The focus of the present paper was to establish a causal relationship between dorsomedial hypothalamic neuropeptide $\mathrm{Y}$ and feeding behavior in rats. However, we would like to emphasize that this study also provides encouraging data in support of using retroviral-mediated therapy for the treatment of obesity and the risk factors associated with diabetes in people. Adeno-associated virus therapy for the treatment of Alzheimer's disease, Parkinson's disease, chronic pain, and other conditions with neuronal targets are currently being assessed in phase I clinical trials and are showing therapeutic potential (Warrington and Herzog, 2006). Because knocking down hypothalamic neuropeptide $\mathrm{Y}$ using adeno-associated virus as well as antagonizing particular neuropeptide $\mathrm{Y}$ receptors reduces food intake and weight gain in rodents, similar manipulations in human subjects could potentially be a treatment for obesity and diabetes. Initial experiments activating or blocking specific neuropeptide $\mathrm{Y}$ receptors in obese human subjects have produced variable results (Erondu et al., 2006; Kamiji and Inui, 2007). Pharmacokinetic weaknesses preventing adequate penetration into the brain after oral administration may explain some of the variability (Kamiji and Inui, 2007). Treatments that enhance pharmacokinetic properties or use central administration, including the retroviral method, might be more efficient in combating obesity and associated risk factors. However, further evaluation of adenoassociated virus-mediated therapy for the treatment of obesity is necessary in both animal and human clinical trials to assess safety concerns and potential side effects.

In sum, Yang et al. (2009) demonstrated that altering neuropeptide Y expression using adeno-associated virusmediated expression resulted in longterm effects on body weight and energy intake. More specifically, an overexpression of neuropeptide $\mathrm{Y}$ increased feeding, whereas knockdown of neuropeptide Y normalized weight gain, food intake, various risk factors for obesity and diabetes, and influenced satiety signaling. The work by Yang et al. (2009) significantly contrib- 
utes to a more complete understanding of the mechanisms and circuitry involved in neuropeptide $\mathrm{Y}$ elicited feeding, and suggests that altering neuropeptide $\mathrm{Y}$ in the hypothalamus may have functional implications for the potential treatment of obesity and diabetes.

\section{References}

Bi S, Robinson BM, Moran TH (2003) Acute food deprivation and chronic food restriction differentially affect hypothalamic NPY mRNA expression. Am J Physiol Regul Integr Comp Physiol 285:R1030-R1036.

Erondu N, Gantz I, Musser B, Suryawanshi S, Mallick M, Addy C, Cote J, Bray G, Fujioka K, Bays H, Hollander P, Sanabria-Bohórquez SM, Eng W, Långström B, Hargreaves RJ,
Burns HD, Kanatani A, Fukami T, MacNeil DJ, Gottesdiener KM, et al. (2006) Neuropeptide Y5 receptor antagonism does not induce clinically meaningful weight loss in overweight and obese adults. Cell Metab 4:275-282.

Gehlert DR (1999) Role of hypothalamic neuropeptide $\mathrm{Y}$ in feeding and obesity. Neuropeptides 33:329-338.

Giraudo SQ, Grace MK, Billington CJ, Levine AS (1999) Differential effects of neuropeptide Y and the mu-agonist DAMGO on 'palatability' vs. 'energy.' Brain Res 834:160-163.

Kamiji MM, Inui A (2007) NPY Y2 and Y4 receptors selective ligands: promising antiobesity drugs? Curr Top Med Chem $7: 1734-1742$

Kovacs P, Hajnal A (2008) Altered pontine taste processing in a rat model of obesity. J Neurophysiol 100:2145-2157.
Levine AS, Kotz CM, Gosnell BA (2003) Sugars: hedonic aspects, neuroregulation, and energy balance. Am J Clin Nutr 78:834S-842S.

MacNeil DJ (2007) NPY Y1 and Y5 receptor selective antagonists as anti-obesity drugs. Curr Top Med Chem 7:1721-1733.

Stanley BG, Kyrkouli SE, Lampert S, Leibowitz SF (1986) Neuropeptide Y chronically injected into the hypothalamus: a powerful neurochemical inducer of hyperphagia and obesity. Peptides 7:1189-1192.

Warrington KH Jr, Herzog RW (2006) Treatment of human disease by adeno-associated viral gene transfer. Hum Genet 119:571-603.

Yang L, Scott KA, Hyun J, Tamashiro KL, Tray N, Moran TH, Bi S (2009) Role of dorsomedial hypothalamic neuropeptide $\mathrm{Y}$ in modulating food intake and energy balance. J Neurosci 29: 179-190. 